\title{
Compilation of small RNA sequences
}

\author{
Jian Gu and Ram Reddy \\ Baylor College of Medicine, Pharmacology Department, Houston, TX 77030, USA
}

\section{ABSTRACT \\ This is an update containing small RNA sequences deposited in GenBank recently. Over four hundred small RNA sequences are available in this and earlier complilations.}

The hard copy print out of this set will be available directly from us (inquiries should be addressed to R.Reddy). These files are also available on GenBank computer. Sequences from various sources covered in earlier compilations (see Reddy,R. Nucleic Acids Res.16: r71; Reddy,R. and Gupta,S. Nucleic Acids Res. 1990 Supplement, 18: 2231 and 1991 Supplement, 19: 2073; Shumyatsky,G. and Reddy,R. Nucleic Acids Res. 1992 Supplement 20: 2159 and 1993,21:3017) are not included in this update. Supported by GM-38320.

U1 snRNA Green alga (Chlamydomonas reinhardtii). Acc. No: X70869. Kis,M., Jakab,G., Pollak,T., Branlant,C. and Solymosy,F. Nucleic Acids Res. 21 (1993) 2255.

U1 snRNA (gene) Nematode (Ascaris lumbricoides). Acc. Nos: L22246, L22248. Shambaugh,J.D., Hannon,G.E. and Nilsen,T.W. unpublished.

U1 snRNA Trematode (Schistosoma mansoni). Acc. Nos: L25914, L25915, L25917, L25919, L25921. El-Meanawy,M., Davis,R.C., Rajkovic,A. and Rottman,F.M. unpublished.

U2 snRNA (gene) Dog (C.Canis). Acc. No: X69482. Verma,M. Unpublished.

U2 snRNA Trematode (S.mansoni). Acc. No: L25918. ElMeanawy,M., Davis,R.C., Rajkovic,A. and Rottman,F.M. unpublished.

U2 snRNA (gene) Sea urchin (Strongylocentrotus purpuratus). Acc. No: M58447. Stefanovic,B.and Marzluff,W.F. Mol. Cell. Biol. 12 (1992) 650-660.

U3 snRNA Amoeba (Tetrahymena thermophila). Acc. No: X71349. Oerum,H., Nielsen,H. and Engberg,J. Nucleic Acids Res. 21 (1993) 2511.

U4 snRNA (gene) Nematode (A.lumbricoides). Acc. No: L22250. Shambaugh,J.D., Hannon,G.E. and Nilsen,T.W. unpublished.

U5 snRNA Maize (Zea mays). Acc. Nos: Z14994, S54006. Leader,D., Connelly,S., Filipowicz,W., Waugh,R. and Brown,J.W. Plant Mol. Biol. 21 (1993) 133-143.

U5 snRNA Nematode (A.lumbricoides). Acc. No: L22251. Shambaugh,J.D., Hannon,G.E. and Nilsen,T.M. unpublished.

U6 snRNA (gene) Nematode (A.lumbricoides). Acc. No: L22252. Shambaugh,J.D., Hannon,G.E. and Nilsen,T.M. unpublished.
U6 snRNA Trematode (S.mansoni). Acc. No: L25920. ElMeanawy,M., Davis,R.C., Rajkovic,A. and Rottman,F.M. unpublished.

U6 snRNA Sea urchin (S.purpuratus). Acc. No: X76389. Sakallah,S.A., Norton,D.R., Zhang,W. and Marzluff,W.F. Biochem. Biophys. Acta. (In press).

U8 snoRNA Frog (Xenopus laevis). Acc. No: L13416. Peculis,B.A. and Steitz,J.A. Cell 73 (1993)1233-1245.

U15 snoRNA Human (H.sapiens). Acc. Nos: L15616 and L15557. Tycowski,K.T., Shu,M.D. and Steitz,J.A. Genes and Dev. 7 (1993) 1176-1190.

U16 snoRNA (gene) Human (H.sapiens). Acc. No: X72205. Fragapane,P., Prislei,S., Mischienzi,A., Caffarelli,E. and Bozzoni,I. EMBO J. 12 (1993) 2921-2928.

U17 snoRNA (also gene) Human (H.sapiens). Acc. Nos: S63850, L16791 and L16792. Kiss,T. and Filipowicz,W. EMBO J. 12 (1993) 2913-2920; Acc. No: L22738. Nag,M.K., Thai,T.T., Ruff,E.A., selvamurugan,N., Kunnimalaiyaan,M. and Eliceiri,G.L. Proc. Natl. Acad. Sci. U.S.A. 90 (1993) 9001-9005.

U18 snoRNA (gene) Frog (X.tropicalis, X.laevis) and Human (H.sapiens). Acc. Nos: X75486-8. Prislei,s., Michienzi,A., Presutti,c., Fragapane.P. and Bozzoni,I. Nucleic Acids Res. 21 (1993) 5824-5830.

snR31 RNA (gene) Yeast (S.cerevisiae). Acc. Nos: X72299, L22433. Dandekar,T. and Tollervey,D. Nucleic Acids Res. 21 (1993) 5386-5390; Balakin,A.G., Schneider,G.S., Corbett,M.S., Ni,J. and Fournier,M.J. Nucleic Acids Res. 21 (1993) 5391-5397.

snR32 RNA (gene) Yeast (S.cerevisiae). Acc. No: L22434. Balakin,A.G., Schneider,G.S., Corbett,M.S., Ni,J. and Fournier,M.J. Nucleic Acids Res. 21 (1993) 5391-5397.

snR33 RNA (gene) Yeast (S.cerevisiae). Acc. No: L22435. Balakin,A.G., Schneider,G.S., Corbett,M.S., Ni,J. and Fournier,M.J. Nucleic Acids Res. 21 (1993) 5391-5397.

E2 snoRNA (gene) Human. Acc. No: L22739. Nag,M.K., Thai,T.T., Ruff,E.A., selvamurugan,N., Kunnimalaiyaan,M. and Eliceiri,G.L. Proc. Natl. Acad. Sci. U.S.A. 90 (1993) 9001-9005.

E3 snoRNA (gene) Human. Acc. No: L22740. Nag,M.K., Thai,T.T., Ruff,E.A., selvamurugan,N., Kunnimalaiyaan,M. and Eliceiri,G.L. Proc. Natl. Acad. Sci. U.S.A. 90 (1993) 9001-9005. 
T3-1 snRNA Amoeba (Tetrahymena thermophica). Acc. No: X66429. Nielsen,H., Orum,H. and Engberh,J. FEBS Lett. 307 (1992) 337-342.

T3-2 snRNA Amoeba (T.thermophica). Acc. Nos: X66430, S41684. Nielsen,H., Orum,H. and Engberh,J. FEBS Lett. 307 (1992) 337-342.

T3-3 snRNA Amoeba (T.thermophica). Acc. Nos: X66431, S41685. Nielsen,H., Orum,H. and Engberh,J. FEBS Lett. 307 (1992) 337-342.

T3-4 snRNA Amoeba (T.thermophica). Acc. Nos: X66432, S41686. Nielsen,H., Orum,H. and Engberh, J. FEBS Lett. 307 (1992) 337-342.

7SL RNA (gene) Crucifer (Arabidopsis thaliana). Acc. Nos: X72228 and X72229. Marques,J., Gualberto,J.M and Palme,K. Nucleic Acids Res. 21 (1993) 3581 .

MCS1 RNA Bacteria (Mycoplasma capricolum). Acc. No: S67588. Ushida,C. and Muto,A. Nucleic Acids Symp. Ser. (1993) 157-158

MCS2 RNA (also gene) Bacteria (M. capricolum). Acc. Nos: S67590/D13063. Ushida,C. and Muto,A. Nucleic Acids Symp. Ser. (1993) 157-158; Nucleic Acids Res. 21 (1993) 2649-2653.

MCS3 RNA (also gene) Bacteria (M.capricolum). Acc. Nos: S67591/D13064. Ushida,C. and Muto,A. Nucleic Acids Symp. Ser. (1993) 157-158; Nucleic Acids Res. 21 (1993) 2649-2653.

MCS4 RNA (also gene) Bacteria (M.capricolum). Acc. Nos: S67596/D13065. Ushida,C. and Muto,A. Nucleic Acids Symp. Ser. (1993) 157-158; Nucleic Acids Res. 21 (1993) 2649-2653.

MCS5 RNA (also gene) Bacteria (M. capricolum). Acc. Nos: S67629/D13066. Ushida,C. and Muto,A. Nucleic Acids Symp. Ser. (1993) 157-158; Nucleic Acids Res. 21 (1993)2649-2653.

MCS6 RNA (gene) Bacteria (M.capricolum). Acc. No: D13067. Ushida,C. and Muto,A. Nucleic Acids Res. 21 (1993) 2649-2653.

MRP RNA (gene) Bovine (Bos taurus). Acc. No: L22578. Dairaghi,D.J. and Clayton,D.A. J. Mol. Evol. 37 (1993) 338.

MRP/7-2 RNA Crucifer (A.thaliana). Acc. Nos: X65942 and S46534. Kiss,T., Marshallsay,C. and Filipowicz,W. EMBO J. 11 (1992) 3737-3746.

Y3 scRNA (gene) Frog (X.laevis). Acc. No: L15431. O'Brien,C.A., Margelot,K., and Wolin,S.L. Proc. Natl. Acad. Sci. U.S.A. 90 (1993) 7250-7254.

Y4 scRNA (gene) Frog (X.laevis). Acc. No: L15432. O'Brien,C.A., Margelot,K., and Wolin,S.L. Proc. Natl. Acad. Sci. U.S.A. 90 (1993) 7250-7254.

Y5 scRNA (gene) Frog (X.laevis). Acc. No: L15433. O'Brien,C.A., Margelot,K., and Wolin,S.L. Proc. Natl. Acad. Sci. U.S.A. 90 (1993) 7250-7254.

Y $\alpha$ scRNA (gene) Frog (X.laevis). Acc. No: L15434. O'Brien,C.A., Margelot,K., and Wolin,S.L. Proc. Natl. Acad. Sci. U.S.A. 90 (1993) 7250-7254. .

RNase P RNA (gene) Bacteria (Bacteroides thetaiotaomicron). Acc. No: L25702. Haas,E.S., Brown,J.W., Pitulle,C. and Pace,N.R. unpublished (1994).
RNase P RNA (gene) Bacteria (Chlorobium limicola). Acc. No: L25703. Haas,E.S., Brown,J.W., Pitulle,C. and Pace,N.R. unpublished (1994).

RNase P RNA (gene) Bacteria (Chlorobium tepidum). Acc. No: L25704. Haas,E.S., Brown,J.W., Pitulle,C. and Pace,N.R. unpublished (1994).

RNase P RNA (gene) Bacteria (Flavobacterium yabuuchiae). Acc. No: L25705. Haas,E.S., Brown,J.W., Pitulle,C. and Pace,N.R. unpublished (1994).

RNase P RNA (gene) Bacteria (Mycoplasma hyopneumoniae). Acc. No: X69982. Svard,S.G., Mattsson,J.G., Johansson,K.E. and Kirsebom,L.A. unpublished.

RNase P RNA (gene) Bacteria (Mycoplasma flocculare). Acc. No: X69983. Svard,S.G., Mattsson,J.G., Johansson,K.E. and Kirsebom,L.A. unpublished.

RNase P RNA (gene) Archaea (Sulfolobus acidocaldarius). Acc. No: L13597. LaGrandeur,T.E., Darr,S.C., Haas,E.S. and Pace,N.R. J. Bacteriol. 175 (1993) 5043-5048.

BC1 scRNA Guinea pig (Cavia porcellus). Acc. No: U01304. Martignetti,J.A. and Brosius,J. Proc. Natl. Acad. Sci. U.S.A. 90 (1993) 9698-9702.

BC1 scRNA Hamster (Microcricetus aureus). Acc. No: U01309. Martignetti,J.A. and Brosius,J. Proc. Natl. Acad. Sci. U.S.A. 90 (1993) 9698-9702.

BC1 scRNA Mouse (Mus musculus). Acc. No: U01310. Martignetti,J.A. and Brosius,J. Proc. Natl. Acad. Sci. U.S.A. 90 (1993) 9698-9702.

BC200 scRNA (also gene) Human (H.sapiens). Acc. Nos: U01305/U01306. Tiedge,H., Chen,W. and Brosius,J. J. Neurosci. 13 (1993) 2382-2390.

Alu scRNA Human (H.sapiens). Acc. Nos: L13704-15. Maraia,R.J., Driscoll,C.T., Bilyeu,T., Hsu,K. and Darlington,G.J. Mol. Cell. Biol. 13 (1993) 4233-4241. 